\title{
Les options de l'aménagement du territoire à la lumière des enjeux environnementaux
}

\author{
Louis Guay ${ }^{1}$ \\ Université Laval
}

\section{Introduction}

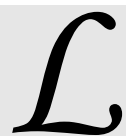
es territoires du Québec ont subi de profonds changements depuis la Seconde Guerre mondiale. Ils ont toujours été l'objet d'interventions humaines soutenues, s'accélérant ou se ralentissant au rythme de l'économie, de la démographie, des technologies et des choix politiques. L'aménagement du territoire s'est développé en trois grandes phases : une phase de poursuite de la construction territoriale, commencée il y a longtemps, favorisée par la

On peut qualifier de manière imagée ces trois périodes de territoires construits, territoires contestés et territoires conciliés. forte croissance économique d'après-guerre et une urbanisation qui s'accélère; une phase, qui se manifeste dès la fin des années 1960, mais qui prend son envol dans les années 1970 et 1980, durant laquelle plusieurs décisions et politiques en aménagement sont contestées; enfin la phase plus récente, produite par les années 1990 et qui se poursuit aujourd'hui, où l'aménagement du territoire est soumis à des idées et pressions qui le conduisent à revoir ses manières de faire et ses choix de politiques.

On peut qualifier de manière imagée ces trois périodes de territoires construits, territoires contestés et territoires conciliés. Le choix de territoires au pluriel est délibéré. Le territoire du Québec est fort varié : la géographie le montre d'emblée. Ensuite, les responsabilités en aménagement du territoire sont largement partagées, pour ne pas dire divisées : les décideurs sont fort différents; les organismes régulateurs des décisions, comme les ministères, les municipalités, sont fragmentés et souvent en concurrence les uns avec les autres. Chaque organisme a ses compétences, ses orientations, ses manières de faire et ses mandataires sociaux, économiques et politiques. Son action porte sur un territoire particulier. Les découpa- ges sont fort nombreux et les usages aussi. Il y a les territoires qu'on habite, ceux qu'on exploite et aménage intensivement (la ville, la campagne agricole, la forêt), ceux qu'on fréquente pour la récréation, le loisir et la découverte, ceux qu'on désire conserver intacts; enfin, quand ils ont été abîmés, ceux qu'on tente de restaurer. Ils sont des milieux de vie, humaine et non humaine, que nous nous partageons. Sur un même territoire, les usages peuvent être variés et des conflits d'usage peuvent surgir. Ceux-ci ne sont pas insolubles mais, pour l'aménagement, ils exigent souvent de longues négociations pour arriver à un partage acceptable entre divers usagers.

Le texte qui suit comprend deux parties qui analysent les voies d'avenir de l'aménagement au Québec. Il débute avec l'examen de l'aménagement à la lumière du développement durable et se termine par la présentation de scénarios d'aménagement qui peuvent se produire.

\section{L'aménagement et le développement durable}

Le rapport de la Commission mondiale sur l'environnement et le développement, Notre avenir à tous, a donné une définition du développement durable qui rend, en matière d'utilisation de l'environnement et des ressources, les générations dépendantes les unes des autres et responsables conjointement de maintenir un environnement de qualité pour la satisfaction des besoins humains présents et à venir.

L'aménagement du territoire a profité de la vague environnementale. Les lois générales sur l'environnement et les audiences publiques sur les impacts des 
projets ont permis de jeter un éclairage neuf sur l'aménagement des territoires. Un lien presque naturel s'est établi entre l'environnement et l'aménagement, du moins en principe. L'aménagement a dû intégrer à ses pratiques les orientations et les normes environnementales. Le cadre législatif a apporté à l'aménagement une rigueur à l'égard de la conservation des écosystèmes qui lui faisait défaut quand ses décisions étaient, comme les politiques régionales et l'expansion urbaine, dominées par le développement. Cependant, l'aménagement et la protection de l'environnement ont, plus d'une fois, divergé sur des projets particuliers et sur le choix des moyens. Les grands projets hydroélectriques nordiques canadiens ont souvent mis en évidence les contradictions entre le développement et son aménagement et la protection de l'environnement.

Le Sommet de Rio de Janeiro de 1992 est venu conclure une intense activité internationale visant une meilleure protection de l'environnement planétaire. Trois conventions cadres sur les changements climatiques, sur la diversité biologique et sur la désertification ont été signées par une forte majorité d'États. Quelles sont les conséquences possibles et souhaitables des conventions de Rio (changements climatiques et diversité biologique pour ce qui concerne le Québec) sur l'aménagement des territoires ?

C'est peut-être avec la politique forestière qu'il faudrait commencer à s'interroger sur les impacts des conventions de Rio sur l'aménagement. En effet, étant donné sa vaste taille, sa propriété publique, le fait que, pour beaucoup de collectivités locales, la forêt est source de valeurs diverses (emplois, récréation, tourisme, services écologiques), une politique d'aménagement devrait débuter par la forêt. La politique et la gestion forestières québécoises sont récemment passées par des modifications de régime et de pratiques qui, malgré les contestations et visions différentes, montrent une ouverture aux idées d'aménagement durable ${ }^{2}$.

La Loi sur l'aménagement et l'urbanisme se préoccupe de planification des territoires habités. Quelles sont les idées et les politiques qui pourraient être mises de l'avant pour un aménagement durable ? Il faut commencer par les villes, là où vivent, selon le dernier recensement, plus de $80 \%$ de la population canadienne, y compris au Québec.

La ville a-t-elle pris à bras le corps le développement durable? Il y a plusieurs raisons pour lesquelles on peut défendre une politique active d'aménagement durable dans les villes. À part le fait qu'elles abritent une forte majorité de la population, les citadins sont généralement plus riches et donc de plus grands consommateurs de ressources de toutes sortes. Grâce à leurs revenus plus élevés, ils commandent plus de matières et d'énergie et, en vertu de leurs modes d'habitation, consomment de larges espaces totalement artificialisés. Cette consommation entraîne des résidus, déchets qui, malgré les mesures de récupération et de recyclage, échouent dans l'environnement. De plus, bien que l'on observe des tendances à la « dématérialisation » et à la « décarbonisation » dans les économies tournées vers l'information et le savoir, il reste que cette économie ne saurait se passer de ressources matérielles: elle consomme de l'environnement, si on peut dire, certes moins que les économies industrielles anciennes pour la même unité de produit intérieur brut, mais elle ne peut prospérer sans base matérielle. Enfin, certains problèmes environnementaux sont concentrés dans les villes et les grandes villes : la pollution atmosphérique touche les grandes villes dominées par l'industrie et par le transport automobile. Or, le secteur des transports est, au Canada, la principale source d'émissions de gaz à effet de serre.

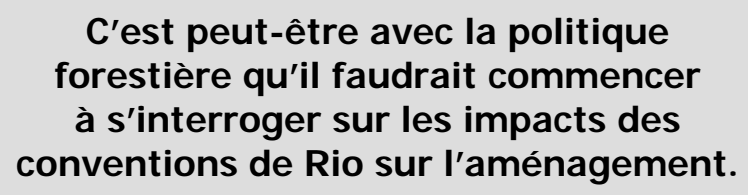

Les solutions proposées semblent aller de soi : réduire le transport privé; augmenter les transports en commun; planifier des villes compactes, sans compter toutes les innovations économes d'énergie qu'on pourrait développer, diffuser et adopter pour améliorer l'efficacité énergétique des bâtiments et réduire les pertes dans les grandes infrastructures urbaines. L'Europe a déjà donné le ton dans ce domaine, mais certaines villes des pays en voie de développement sont aussi exemplaires ${ }^{3}$. Or, on ne semble pas mesurer suffisamment les écueils qu'il faut franchir pour s'approcher d'une ville durable qui réduit son empreinte écologique ${ }^{4}$. En premier lieu, modifier radicalement la forme urbaine et améliorer les infrastructures urbaines nécessitent, d'une part, des investissements considérables et, d'autre part, une politique d'aménagement 
soutenue sur le long terme. En second lieu, le transport interurbain, soit pour le loisir et la récréation, soit pour le transport des produits dans une économie continentale et mondiale de plus en plus intégrée, échappe à la politique d'aménagement urbain. On peut souhaiter une révolution des technologies des transports, mais elle se fait attendre. En troisième lieu, l'automobile occupe une place de premier choix dans les économies industrielles avancées. On l'a largement adoptée, on s'y est fait, y compris en planification urbaine, de telle sorte qu'opérer un renversement de la vapeur apparaît du domaine du rêve plus que de la réalité. Et pourtant, on sent qu'il faut agir sur ce front. La résistance du consommateur citadin risque d'être forte. En effet, une enquête comparative montre que, si les gens sont prêts à payer un peu plus cher les produits et à verser plus de taxes et d'impôts pour mieux protéger l'environnement, ils sont moins bien disposés à réduire significativement l'utilisation de l'auto en l'absence de bonnes solutions de rechange. Enfin, pour encourager le passage du transport privé au transport public urbain, celui-ci a probablement besoin d'une révolution technologique, améliorant notamment le confort des passagers.

\section{On s'attarde beaucoup à la création des aires protégées qui représente très certainement le pilier central d'une politique de la biodiversité.}

La Convention de Rio sur la diversité biologique vise à protéger la vie sous toutes ses formes et à toutes ses échelles : diversité génétique, spécifique, écosystémique et paysagère. Elle ne manque pas d'ambition, mais sa mise en œuvre risque d'être plus ardue. D'une part, on touche directement aux ressources quand on désire protéger la biodiversité. D'autre part, les acteurs n'ont pas tous la même conception de la biodiversité à sauvegarder, voire à augmenter ${ }^{5}$. En outre, on ne s'entend pas toujours sur les moyens de la conserver et sur les principes qui doivent guider son utilisation, surtout dans un contexte de commerce international. Enfin, il faut tenir compte de la révolution biotechnologique et pharmaceutique qui fonde la prospérité de l'industrie qu'elle nourrit sur l'accès au capital génétique et donc à la biodiversité partout où elle se trouve, notamment dans les zones tropicales. Or, l'accès libre aux ressources génétiques est un des aspects les plus controversés qui a surgi dans la pré- paration et la mise en œuvre de la Convention sur la diversité biologique.

Comment l'aménagement peut-il répondre à ce nouveau contexte? Il faut préciser que c'est le gouvernement fédéral qui a signé la Convention sur la diversité biologique, alors que ce sont les provinces qui gèrent la plupart des ressources naturelles, et donc les espaces de biodiversité. Il existe bien une stratégie fédérale et une stratégie québécoise sur la biodiversité, et le territoire canadien consacré aux aires protégées s'est accru depuis la signature de la Convention ${ }^{6}$. Toutefois, ces stratégies sont en attente d'être prises au bond et intégrées aux pratiques territoriales diverses. Comment l'aménagement urbain peut-il faire sa part pour protéger, maintenir, augmenter la biodiversité urbaine? Il n'est pas impensable que la ville entreprenne une sorte de révolution verte dans laquelle elle ferait plus de place aux espaces verts, aux parcs, à la protection des milieux humides, à la préservation des espèces qui y vivent et consommerait moins de ressources et d'espace. Mais sa contribution sera toujours limitée: une ville comporte peu de nature, même si l'aménagement peut en accroître les superficies et la diversité.

C'est du côté d'autres politiques du territoire qu'on doit se tourner. La foresterie, l'agriculture, la pêche ont toutes un rôle crucial à jouer dans la mise en œuvre de la Convention. On s'attarde beaucoup à la création des aires protégées qui représente très certainement le pilier central d'une politique de la biodiversité, mais, en désirant augmenter les superficies intégralement ou largement protégées, on risque de devoir affronter des intérêts socio-économiques prêts à défendre leur conception et leur usage des territoires. Par exemple, en territoire forestier, les écologistes, professionnels et militants ont demandé que soit tracée une limite nordique d'exploitation forestière. Si l'idée a été en principe acceptée, la ligne n'est toujours par tirée. Et on peut s'attendre à une controverse publique lorsque la décision gouvernementale sera prise, les uns la trouvant trop haute, les autres trop basse. Si l'on décide d'augmenter les aires protégées en territoire forestier qui avait, jusqu'alors, été exploité pour le bois, on risque de rendre désuètes des économies locales entières et d'attiser ainsi les conflits sociaux, sans parler des difficultés de reconversion des compétences personnelles et communautaires vers des fonctions tout à fait différentes. 
Enfin, il faudrait très certainement amorcer une profonde réflexion sur le territoire agricole et sur son rôle dans la protection de la biodiversité. Une agriculture encore plus intensive, fondée sur l'utilisation à grande échelle des plantes transgéniques encore plus productives et plus résistantes aux parasites, est-elle la voie du succès ou conduit-elle à de plus grands risques environnementaux ? La controverse sur les organismes génétiquement modifiés qui s'est développée en plusieurs pays est annonciatrice de nuages lourds à l'horizon si l'on suit cette voie à train d'enfer. Ce qui est le plus inquiétant pour les producteurs et les décideurs publics, c'est que, dans ce domaine, les opposants peuvent protester de différentes manières : en élevant la voix mais aussi par des choix d'achat qui fuient les produits qui ne sont pas étiquetés «sans OGM ».

\section{Les voies de l'avenir : des questions à débattre}

Nul doute que le grain semé par la CMED (Commissison mondiale sur l'environnement et le développement) - et l'Union mondiale pour la nature, le World Wildlife Fund et le Programme des Nations Unies pour l'environnement (PNUE) avant elle - a germé et que le développement durable est une plante qui résiste bien aux intempéries, même si elle crôit lentement. Les gouvernements, les entreprises, les municipalités se réclament tous du développement durable et, dans les secteurs d'activités concernés, de l'aménagement durable des milieux. Toutefois, rien ne garantit que l'avenir du développement durable soit assuré, en partie parce qu'il veut dire différentes choses à différentes personnes, et en partie parce que la voie pour y arriver est semée d'embûches et d'obstacles. Comme la modernité avant lui, le développement durable ouvre la porte vers l'inconnu et l'imprévu. La modernité est sûrement devenue bien autre chose que ce qu'en imaginaient ses premiers concepteurs, et on peut se demander si l'on sera en mesure de reconnaître le développement durable quand on sera à sa porte et sous son toit.

La section qui suit sera forcément plus spéculative. L'aménagement est soumis à des pressions nouvelles, notamment à cause de la mondialisation économique, politique et culturelle. La mondialisation, aux yeux de ses théoriciens les plus avertis, comme Giddens, n'est pas une fuite vers le haut, comme pour se libérer des limites et des contraintes locales, mais une articulation plus étroite et plus solide entre l'échelle mondiale et les échelles nationales, régionales et locales pour une bonne partie des activités humaines et de leurs incidences. Les problèmes écologiques globaux, par exemple, prennent leur source dans des gestes et des décisions locaux mais se répercutent, tels les changements climatiques, à de très larges échelles temporelles et spatiales.

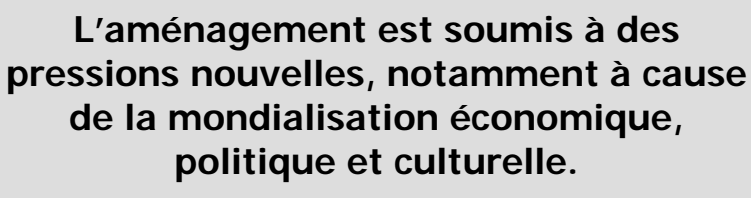

Que sera, dans ce contexte de changements et de tendances nouvelles, l'aménagement durable des villes et des régions, des territoires habités et utilisés ? L'aménagement n'est pas en manque de défis et de questions à débattre, dont voici un petit échantillon.

\section{Le développement et l’aménagement}

À tout seigneur tout honneur, quel type de développement apparaît à la fois raisonnable et souhaitable compte tenu des valeurs sociales dominantes et des connaissances acquises sur l'environnement? Les sociétés ne peuvent changer tout d'un coup et redéfinir leurs pratiques du jour au lendemain. En matière de développement, qui a presque toujours été une idée contestée, même quand elle était largement partagée, on est en droit de se demander si la route prise et suivie jusqu'ici mène au bon endroit. On a appris à compter sur les progrès de la science et de la technologie pour produire le développement, mais celui-ci est plus large; au cœur du développement, on trouve des institutions sociales qui le favorisent et un «culture qui importe », tournée vers le progrès matériel.

Les problèmes qui se posent à l'aménagement des villes et des régions au Québec ne lui sont certes pas uniques. Devant l'exode des jeunes des régions, celles-ci se demandent que faire. Les grandes villes continuent de s'étendre et une "ex-urbanisation » limitée vers quelques satellites relativement proches s'y produit. En prenant comme indicateur les villes moyennes (tableaux 1 et 2), on peut mesurer les tendances opposées au sein du système urbain du Québec. 


\section{Tableau 1 - Villes moyennes en forte croissance (aires de recensement : moins de 100000 habitants)}

\begin{tabular}{lc} 
Ville & $\begin{array}{c}\text { Variation de population } \\
\text { en \%, 1996-2001 }\end{array}$ \\
\hline Saint-Georges & 5,8 \\
Magog & 5,6 \\
Drummondville & 5,1 \\
Joliette & 4,2 \\
Saint-Jean-sur-Richelieu & 4,1 \\
\hline
\end{tabular}

Source : Statistique Canada, Chiffres de population et de logements, Canada, provinces et territoires, régions métropolitaines de recensement et agglomérations de recensement, recensements de 2001 et de 1996. Ottawa, 2003.

Plusieurs villes moyennes en fort déclin sont «périphériques »; plusieurs villes moyennes en forte croissance se trouvent, mais pas toutes, dans le giron de la métropole de Montréal. Les politiques de développement et d'aménagement seront assez différentes dans ces deux situations. Et si la métropolisation se poursuit, très fortement concentrée dans la région de Montréal, dans la région d'Ottawa-Gatineau et, à un moindre degré, dans celle de Québec (tableaux 3 et
4), le système spatial québécois risque de se découpler davantage, c'est-à-dire que les régions dynamiques poursuivent une politique propre et une trajectoire ascendante, alors que les régions en déclin tentent de mener une autre politique sur une trajectoire descendante ${ }^{7}$.

\section{Tableau 2 - Villes moyennes en déclin (aires de recensement : moins de 100000 habitants)}

\begin{tabular}{|lc|}
\hline Ville & $\begin{array}{c}\text { Variation de population } \\
\text { en \%, 1996-2001 }\end{array}$ \\
\hline Baie-Comeau & $-9,0$ \\
Rouyn-Noranda & $-7,1$ \\
Thetford Mines & $-5,2$ \\
Matane & $-5,1$ \\
Sorel-Tracy & $-4,8$ \\
Shawinigan & $-4,3$ \\
La Tuque & $-4,2$ \\
\hline
\end{tabular}

Source : Statistique Canada, Chiffres de population et de logements, Canada, provinces et territoires, régions métropolitaines de recensement et agglomérations de recensement, recensements de 2001 et de 1996. Ottawa, 2003.

\section{Tableau 3 - Données sur l'évolution des régions métropolitaines de recensement (RMR), en milliers}

\begin{tabular}{lccccc} 
RMR & $\mathbf{1 9 6 6}$ & $\mathbf{1 9 8 6}$ & $\mathbf{2 0 0 2}$ & $\begin{array}{c}\text { \% } \\
\text { variation } \\
\mathbf{1 9 9 6 - 2 0 0 1}\end{array}$ & $\begin{array}{c}\text { \% né à } \\
\text { l'étranger } \\
\text { en 2001 }\end{array}$ \\
\hline Montréal & 2437 & 2921 & 3548,8 & 3,0 & 18,4 \\
Ottawa-Hull (Gatineau*) & 495 & 819 & 1128,9 & 6,5 & 17,6 \\
Québec & 413 & 603 & 697,8 & 1,6 & 2,9 \\
Chicoutimi-Jonquière (Saguenay*) & 109 & 158 & 156,9 & $-3,4$ & 0,9 \\
Sherbrooke & 78 & 130 & 156,5 & 2,8 & 4,6 \\
Trois-Rivières & 95 & 130 & 141,4 & $-1,7$ & 1,5 \\
\hline
\end{tabular}

* Nouvelle appellation depuis 2002.

** Gatineau seulement

Source: Statistique Canada, Population des régions métropolitaines de recensement; Un profil de la population canadienne : où vivons-nous? et Le Canada en statistiques. Proportion de personnes nées à l'étranger, régions métropolitaines de recensement. Ottawa, 2003. 


\section{Tableau 4 - Concentration métropolitaine en 2001 : population en milliers}

$\begin{array}{lc}\text { Population du Québec } & 7237,0 \\ \text { Population des 6 RMR* (Gatineau =258,0) } & 4918,4 \\ \text { Population non-RMR } & 2318,2 \\ \text { RMR/ population du Québec } & 67,9 \% \\ \text { RMR Montréal/population du Québec } & 48,4 \% \\ \text { RMR Montréal/toutes les RMR } & 71,4 \% \\ & \\ \text { * Sans Ottawa. } & \\ \text { Source : Statistique Canada (2003). Population des régions métropolitaines, } \\ \text { et Un profil de la population canadienne : où vivons-nous? Ottawa. }\end{array}$

L'urgence de politiques d'équilibre régional peut revenir à l'ordre du jour, mais les décideurs pourraient aussi opter pour une intervention moins lourde dans les régions en déclin, acceptant ainsi des migrations plus fortes. On peut envisager une agriculture et une foresterie différentes pour ces régions, fondées sur la ferme familiale, l'agriculture biologique à petite échelle et la foresterie communautaire, par exemple, qui maintiendraient et augmenteraient l'emploi local et la vitalité régionale. On peut toutefois se demander si, dans un contexte de mondialisation, la productivité de ces entreprises pourra concurrencer avec les grands acteurs économiques internationaux et nationaux.

\section{La gouvernance territoriale}

Quelle unité territoriale de gouvernance significative doit-on choisir en aménagement? Cette question n'est pas sans importance. En effet, on a récemment profondément modifié les structures municipales, choisissant le regroupement en des entités plus larges et plus peuplées ${ }^{8}$. Celles-ci sont acquises et il n'est pas question de retourner en arrière. Même si elles n'ont pas principalement été créées pour favoriser une meilleure politique d'aménagement, la planification territoriale n'a pas été absente du débat que la politique de fusion a provoqué. La responsabilité d'aménager le territoire (habité, faudrait-il toujours ajouter) qui est dévolue aux municipalités ne concorde pas bien avec les nouvelles conceptions des territoires et la nécessité d'être gérés sur des bases plus écologiques que purement historiques, politiques et administratives. On vante la gestion écosystémique, la gestion par bassin versant, mais la réflexion et la pratique sont peu avancées. Toutefois, avec la nouvelle politique de l'eau, un pas en avant a été fait vers une gestion territoriale fondée sur le bassin versant qui doit amener tous les acteurs locaux à coordonner leurs interventions et à établir des plans de l'eau sur une unité territoriale géographiquement significative. Ces plans de l'eau deviendront-ils la base de schémas d'aménagement élargis ? Quant à l'approche écosystémique, elle a énormément de sens dans une gestion de l'environnement intégrée, mais aucune politique d'ensemble n'a émergé et les expériences en cours ne se font, même au sein des aires protégées, que sur des unités territoriales limitées ${ }^{9}$. Il faut souvent penser plus grand dans la gestion des territoires.

Il restera toujours le cas des grandes régions urbaines qui se définissent mal en termes écologiques et géographiques. D'abord, parce que leurs frontières reculent constamment avec l'étalement; ensuite, parce que le poids du passé y est très lourd - les municipalités à majorité anglophone ou à forte proportion d'anglophones de l'Île de Montréal ont voté, pour des raisons défendables certes, mais au risque d'affaiblir une région métropolitaine qui ne se classe pas parmi le peloton de tête économique en Amérique du Nord ${ }^{10}$, pour se détacher de la nouvelle ville unifiée et conserver leur autonomie -; enfin, parce que les régions urbaines sont des "écosystèmes » artificiels, presque totalement construits et inventés par la raison et l'imagination humaines. Elles ont néanmoins besoin de ressources naturelles et de biens environnementaux comme l'eau, ce qui ne les exclut pas entièrement d'une participation à une gestion territoriale établie selon un découpage et des limites géoécologiques. 
L'aménagement des territoires doit-il expérimenter de nouveaux modes de gestion comme les régimes d'environnement et de ressources ? L'idée de régime est apparue dans les recherches sur la gestion des ressources communes. Peut-on, avec profit, importer cette innovation institutionnelle en aménagement au Québec ? Est-il pensable que, pour atteindre des objectifs de développement durable, naissent des régimes d'environnement et d'aménagement localisés, adaptés aux caractéristiques particulières des territoires, mais intégrant tous les aspects territoriaux et écologiques ? Les comités de bassin semblent être un pas dans cette direction. Les régimes de ressources, s'ils voient le jour, ne pourront qu'être régionaux à cause de la grande diversité des milieux naturels du Québec. Les acteurs, les décideurs et les organismes locaux et régionaux devront apprendre à collaborer, partager des informations, se définir des objectifs, mettre en commun des ressources et exercer une surveillance sur leurs propres décisions. Les gouvernements supra municipaux qui sont bien implantés, la variété des formes de concertation et de forums régionaux qui se sont développés sont certes un bon premier pas dans la bonne direction, mais ils ne forment pas encore des institutions, ou régimes, d'intervention forte sur les enjeux environnementaux et territoriaux, dont les contours épousent mal les découpages administratifs et les modes de gestion en place.

Et puis, si les acteurs locaux et régionaux acquièrent ressources, responsabilités et expérience, les régimes renforceront la décentralisation territoriale tant souhaitée, avec le risque toutefois que de grandes disparités apparaissent entre eux s'ils ne sont pas coordonnés par une autorité supérieure.

\section{Est-il pensable que, pour atteindre des objectifs de développement durable, naissent des régimes d'environnement et d'aménagement localisés, adaptés aux caractéristiques particulières des territoires, mais intégrant tous les aspects territoriaux et écologiques ?}

\section{Les limites des territoires}

Les territoires possèdent-ils des limites naturelles qu'on ne peut dépasser au risque de les rendre tout à fait impropres à l'usage humain ? Cette question diffi- cile est amplement débattue en écologie où il est généralement admis que les écosystèmes ont des capacités de charge limite qui ne peuvent être dépassées. Cependant, cette thèse repose beaucoup sur des écosystèmes de petite ou de moyenne échelle, et on sait encore trop peu de choses sur la réaction des grands complexes écologiques, sans parler de toute la biosphère, à des changements induits par les pratiques humaines, bien que le signal du réchauffement climatique nous en donne une certaine idée. L'histoire environnementale montre que les humains ont été en mesure de modifier profondément et irréversiblement de grandes régions écologiques, mais elle ne dit pas où se trouve la ligne des limites de la terre entière à ne pas franchir.

Si les territoires possèdent des limites naturelles et objectives, la planification territoriale doit, pour réussir, se muter en "planification écologique », c'est-àdire décider et agir en fonction des équilibres et des contraintes des milieux. Or, celle-ci peut adopter deux perspectives. La première, la planification écologique forte, repose sur la conviction - ou la connaissance que la planète Terre est, du point de vue des ressources, un système fini et qu'il faut se plier aux limites naturelles. Par conséquent, il importe de planifier l'utilisation des territoires pour en réduire la consommation et, dans certains cas plus extrêmes, de diminuer la population humaine pour atténuer substantiellement les impacts des activités humaines sur la nature. Cette perspective se marie bien avec une position éthique bio ou écocentrique qui conçoit l'espèce humaine comme une autre espèce parmi d'autres soumises aux mêmes lois naturelles. La seconde adopte une position nuancée et plaide pour une planification écologique douce. En effet, comme nos connaissances sur les écosystèmes et sur leurs limites sont incomplètes et incertaines, comme il est aussi difficile de prévoir les changements technologiques radicaux et les capacités humaines à s'adapter aux changements, et comme il n'est pas toujours aisé d'interpréter avec exactitude les signaux sur l' « état de santé » de l'environnement, il faut agir avec prudence et laisser la porte ouverte aux nouvelles connaissances. Il serait, dans cette perspective, hasardeux de s'engager dans la voie préconisée par la première option, comme il est tout autant présomptueux de penser que la biosphère regorge de ressources illimitées.

Ces questions rejoignent les débats sur le rôle et la place du développement durable dans la décision en 
aménagement. Elles sont cependant un peu différentes, car elles nous amènent aussi à réfléchir sur la place des humains dans la nature.

\section{L’aménagement et la participation citoyenne}

Quel type de participation citoyenne doit-on mettre en place? En matière d'environnement et d'aménagement, de considérables progrès ont été accomplis. Les pratiques, les expériences et les compétences se sont rapidement développées. La gestion forestière, par exemple, est embarquée dans une vaste expérience de consultation publique dont on mesure encore mal les effets, positifs et négatifs. L'évaluation environnementale des projets et l'examen public des impacts a acquis au Canada ses lettres de noblesse, même si les mécanismes ne sont pas parfaits ${ }^{11}$. L'aménagement, dans le cadre de la loi 125, est soumis à la consultation publique. Mais tous les problèmes ne sont pas pour autant réglés. Dans un monde social plus diversifié et mieux instruit, dans un univers où les problèmes sont plus complexes, comportant de nombreuses incertitudes et dont les enjeux sont élevés, comme les changements climatiques, la protection de la biodiversité, les risques des plantes transgéniques, qui ont tous des incidences territoriales, la participation citoyenne, c'est-à-dire d'une plus grande diversité d'acteurs sociaux et de perspectives à la décision publique, devient de plus en plus nécessaire ${ }^{12}$. La gouvernance institutionnelle est appelée à changer.

On est souvent frappés que, en aménagement, les gens participent assez largement aux plans de quartiers mais très peu, sauf les experts, aux grands schémas d'aménagement, Or, si les premiers visent l'amélioration de l'habitat immédiat et des conditions de vie locales, les seconds sont très déterminants pour l'avenir de toute une collectivité régionale. Les décisions stratégiques concernant l'emplacement des pôles d'emploi, des pôles de services et de commerce, les décisions sur les grands réseaux techniques urbains, les politiques de réseaux verts et de parcs publics, qui façonnent l'avenir et la forme de toute une agglomération, ont des conséquences à long terme considérables sur la vie de tous. Le paradoxe (peu de participation sur les grands enjeux, alors qu'ils touchent sur le long terme tout le monde, mais plus de participation sur les enjeux locaux, alors qu'ils sont limités dans le temps et l'espace) ne cesse de surprendre. Si la participation à la gouvernance locale et régionale doit se développer, il faut tenter de résoudre ce paradoxe. On peut se demander si des méthodes nouvelles visant à favoriser la participation citoyenne, comme les conférences de consensus, les jurys de citoyens, les forums par Internet, les sondages délibératifs, qui ont été expérimentés sur des enjeux sociotechniques controversés comme les OGM, ne pourraient pas être plus fréquemment utilisées en aménagement. Enfin, un des problèmes souvent rencontrés par les participants est la qualité et l'accessibilité de l'information. On se plaint que l'information soit trop spécialisée et semble chercher à faire passer le message que les experts savent et, si les gens savaient, ils en arriveraient aux mêmes conclusions qu'eux. Si la différenciation sociale et le monde moderne ont accentué les spécialisations et l'expertise, des auteurs se sont demandé s'il est sage de faire reposer les grandes décisions publiques sur la seule expertise. En proposant l'idée du " profane expert» ou du citoyen expert, ils tentent de tenir compte que les savoirs sont, justement en vertu d'une modernité avancée plus réflexive sur elle-même, pour reprendre l'expression de Giddens, très largement distribués. Selon eux les savoirs pertinents à une décision publique sont multiples; les valeurs sociales qui guident les décisions, encore plus; et puis, le public est mieux informé et plus éduqué : il peut poser de bonnes questions, contribuer à cadrer les problèmes et les solutions et entrevoir les conséquences des décisions. Par conséquent, sa contribution doit être recherchée non pas tant pour éviter les réactions négatives, mais pour alimenter la discussion et éclairer la décision.

\section{Quel type de participation citoyenne doit-on mettre en place ?}

Dans une telle conception de la participation citoyenne, il irait de soi que les gens soient le plus tôt possible associés au processus de décision et que leurs questions, préoccupations et contributions soient mieux représentées dans la décision finale. C'est une voie que la démocratie et l'aménagement pourront être appelés à adopter dans l'avenir. Le choix des questions d'aménagement et d'environnement à discuter publiquement est étroitement lié à l'élargissement et à l'approfondissement de la participation citoyenne. En effet, les élus et les professionnels ont actuellement l'initiative des débats publics. Il arrive que la mobilisation populaire prenne les devants, mais la plupart des questions sont proposées à l'examen 
public dans le cadre des activités des pouvoirs publics. Ceux-ci réagissent à la pression publique, qu'elle soit exercée par les médias, les intérêts économiques ou les mouvements sociaux et associatifs. Les administrations conservent néanmoins le contrôle des processus de consultation : sur quoi les consultations vont-elles porter; quand et comment se feront-elles; quel est l'échéancier? Or, dans un monde social plus complexe et varié, on peut faire l'hypothèse que la démocratie et la décision collective seraient mieux servies si l'initiative des débats était mieux répartie. Cependant, amorcer un débat n'est pas tout; encore faut-il s'entendre sur comment le conduire.

\section{Les exemples ne manquent pas qui mettent en évidence la difficulté d'arriver à des choix collectifs avantageux pour tous.}

On peut reprocher aux débats sur les politiques publiques de n'être qu'un jeu dans lequel des groupes d'intérêt particuliers, bien structurés et aux préférences bien définies, tentent de faire pencher en leur faveur, par la mise en œuvre des ressources supérieures dont ils disposent, la décision publique. Le forum public se transforme alors en arène où l'emportent ceux qui peuvent mobiliser le plus de ressources matérielles, politiques, cognitives. Les théoriciens de la démocratie de la délibération ont proposé, plutôt souhaité, que les institutions se donnent des principes qui évitent que la décision publique soit prise au piège des plus forts. Ces penseurs défendent l'idée de la valeur intrinsèque de tout participant à une délibération. Le but de celle-ci est que, dans les choix collectifs, le meilleur argument l'emporte et fasse consensus. Bien que cette théorie pèche parfois par excès d'idéalisme, une réflexion sur les principes et les modalités de la délibération et sur ses capacités d'application aurait sa place en aménagement.

Les voies de l'avenir ne sont toutefois pas complètement dessinées. Les systèmes urbains et régionaux, les territoires et l'environnement sont soumis à des forces souvent contradictoires. La réconciliation entre développement et environnement est difficile à réussir; les équilibres régionaux, difficiles à atteindre; l'environnement est sujet à des demandes opposées qui attisent les conflits d'usage; la ville compacte s'oppose à la ville étalée : si la première était visée par les décisions publiques, elle irait à l'encontre des choix individuels dominants pour un habitat relâché. Les exemples ne manquent pas qui mettent en évidence la difficulté d'arriver à des choix collectifs avantageux pour tous.

\section{Les options de l'aménagement}

Il est possible, en s'appuyant sur les enjeux et les problèmes actuels et sur le passé récent dans la planification du territoire, d'envisager trois scénarios aménagistes qui peuvent se réaliser ou dominer les pratiques et les décisions dans un avenir rapproché (tableau 5). Ces scénarios, ou options, sont : la poussée « développementaliste » (néologisme formé à la manière de la dérivation de environnementaliste à partir d'environnement); le repli réglementaire; le pari du développement durable. Chaque scénario est défini par les variables suivantes: les conditions et le contexte de chaque scénario, ce qui le fait naître ou l'encourage; les finalités propres à chacun; les moyens de la mise en œuvre; les acteurs décisionnels clés; le rôle des planificateurs et des savoirs; le processus de décision et de participation; enfin, puisque les effets pervers et les conséquences inattendues ne sont jamais exclus, les horizons négatifs qui peuvent émerger.

Le premier scénario ressemble à la poursuite de la politique de développement qui s'est graduellement implantée depuis la Seconde Guerre mondiale. La mondialisation, l'assainissement des finances publiques, les besoins qui restent à combler, notamment dans les régions et les villes aux prises avec la fermeture des entreprises traditionnelles et avec un taux de chômage élevé, créent un environnement favorable à la reprise d'une politique de développement à tout prix. La croissance économique apparaît comme la principale, sinon la seule, source de bien-être. Le développement doit toutefois se faire en tenant compte de l'internationalisation croissante des économies. Les moyens d'une politique publique de développement devront, en conséquence, s'ajuster à soutenir les entreprises innovantes, performantes et exportatrices. Les acteurs économiques privés dominent la scène, plaidant pour des contrôles et une réglementation facilitant les décisions privées et non nuisibles. Les planificateurs jouent un rôle secondaire, appuyant le développement par la production et la répartition spatiale de biens publics, comme des infrastructures 
adéquates. La participation et la consultation publiques sont peu recherchées, car elles sont perçues comme un frein au développement. Deux effets négatifs importants peuvent découler d'un tel scénario. En premier lieu, les impacts sur l'environnement, mesurés par la pollution, l'épuisement des ressources, risquent, en l'absence de changements technologiques majeurs, de s'accroître considérablement. À titre d'exemple, la reprise de la croissance économique canadienne depuis une dizaine d'années a fait augmen- ter les émissions de gaz à effet de serre, laissant croire que le Canada ne pourra atteindre à temps les objectifs qui sont fixés par la ratification du Protocole de Kyoto. En second lieu, les villes et les régions dynamiques peuvent accumuler une bonne part des avantages et des fruits du développement, laissant dans l'ombre les villes et les régions moins bien adaptées, pour toutes sortes de raisons structurelles, historiques, géographiques et institutionnelles, à la mondialisation et à l'économie du savoir.

\section{Tableau 5 - Trois scénarios d'aménagement}

\begin{tabular}{|c|c|c|c|}
\hline Scénarios/Composantes & $\begin{array}{c}\text { La poussée } \\
\text { développementaliste }\end{array}$ & Le repli réglementaire & $\begin{array}{c}\text { Le pari du } \\
\text { développement durable }\end{array}$ \\
\hline Finalités & $\begin{array}{l}\text { Croissance, source de bien- } \\
\text { être; insertion mondiale }\end{array}$ & $\begin{array}{l}\text { Réguler les usages; éviter } \\
\text { les crises }\end{array}$ & $\begin{array}{l}\text { Protection et conservation } \\
\text { de l'environnement; } \\
\text { restauration des milieux } \\
\text { dégradés; usage } \\
\text { soutenable des ressources }\end{array}$ \\
\hline Acteurs décisionnels clés & $\begin{array}{c}\text { Acteurs privés et acteurs } \\
\text { économiques; appui public } \\
\text { ciblé }\end{array}$ & $\begin{array}{l}\text { Acteurs publics et acteurs } \\
\text { juridiques }\end{array}$ & $\begin{array}{l}\text { Acteurs diversifiés : élus, } \\
\text { professionnels et résidents } \\
\text { locaux et régionaux }\end{array}$ \\
\hline Planificateurs et savoirs & $\begin{array}{l}\text { Aménagiste-entrepreneur } \\
\text { public; savoir de gestion et } \\
\text { de prévision économiques }\end{array}$ & $\begin{array}{l}\text { Aménagiste de contrôle; } \\
\text { savoir normatif }\end{array}$ & $\begin{array}{l}\text { Aménagiste-éducateur, } \\
\text { facilitateur, négociateur; } \\
\text { savoir écologique et } \\
\text { savoirs sociaux }\end{array}$ \\
\hline Horizons négatifs possibles & $\begin{array}{l}\text { Cumul des avantages et } \\
\text { polarisation interrégionale; } \\
\text { milieux dégradés }\end{array}$ & $\begin{array}{l}\text { Fatalisme et conser- } \\
\text { vatisme, repli sur les } \\
\text { intérêts privés }\end{array}$ & $\begin{array}{l}\text { Mise en œuvre locale; } \\
\text { repli localiste; utopie } \\
\text { décourageante; défection }\end{array}$ \\
\hline
\end{tabular}


Le second scénario est une menace permanente en aménagement. Puisque l'aménagement s'occupe pour une bonne part de biens publics, comme l'espace et la qualité de l'environnement, la tentation est souvent forte de se rabattre sur la réglementation, surtout dans un contexte de fort développement où les décisions privées peuvent avoir de nombreux effets négatifs. Dans un tel scénario, la logique d'intervention est de limiter les nuisances de toute nature et de conserver l'espace commun disponible à une diversité d'activités en atténuant les conflits potentiels. Pour l'aménagement, il s'agit de réguler les usages conflictuels des territoires et d'éviter les crises. La réglementation, de préférence centralisée pour assurer la cohérence et l'équité, est l'outil de prédilection, qui peut à terme nuire à ce qui la fait naître, c'est-à-dire la voie du développement. Les acteurs publics, surtout les juristes, dotés d'un savoir élaboré par la diversité des cas qui se présentent à eux, jouent un très grand rôle, bien que leurs fins et leurs moyens soient limités. Le planificateur semble se cantonner dans un rôle de recueillir les doléances, d'observer les nuisances et d'appliquer les règlements. Un tel scénario peut conduire à une grande lourdeur de gestion des territoires et décourager les initiatives. Conservatisme et fatalisme peuvent l'emporter, entraînant dans leur foulée un repli sur les intérêts privés et une défense féroce de ceux-ci.

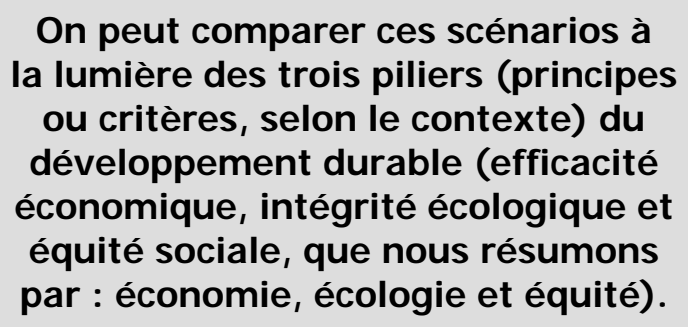

Le dernier scénario est un défi, lancé notamment pas la CMED. La prise de conscience de la fragilité de l'environnement, la montée en force des valeurs écologiques, une meilleure connaissance des impacts des activités humaines sur les milieux, tout cela concourt à rendre le développement durable attrayant et l'une des meilleures solutions aux problèmes de gestion territoriale. La finalité d'un tel scénario est de croire et de faire la preuve qu'il est possible de réconcilier l'économie, l'écologie et l'équité (la règle des trois E). Les moyens à explorer doivent être ambitieux et novateurs : des changements institutionnels, technolo- giques et comportementaux sont, comme le pense la CMED, nécessaires et réalisables. La politique du territoire doit être à la fois intégrée et régionalisée dans, par exemple, des régimes d'environnement et d'aménagement localisés. Les acteurs, insérés dans des processus de forte participation à toutes les étapes, sont diversifiés et nombreux; ils doivent cultiver la communication et l'art de délibérer. Le planificateur, l'aménagiste est un éducateur, un facilitateur, un animateur. Il peut contribuer par ses connaissances et son expertise à la prise de décision, mais il n'a pas le monopole du savoir. Ce scénario, comme les deux autres, peut comporter des effets pervers et ouvrir sur des horizons peu souhaitables. Ce pari repose sur des conditions sociales souvent peu réalistes. D'abord, une grande homogénéité dans la prise de conscience que l'option du développement durable est la seule raisonnable. Or, on sait que le développement durable, s'il fait large consensus sur sa définition, est loin de le faire sur les moyens de le réaliser Les mesures pour mieux protéger l'environnement sont fortement contestées, débattues et parfois battues. Ensuite, si les moyens pour l'atteindre ne sont pas partagés, la tentation est forte que des groupes, des régions pensent localement et agissent localement au détriment d'une coordination territoriale élargie et d'une conception globale du territoire et de son aménagement. On peut ainsi entrevoir des régions qui s'achemineraient dans la voie du développement durable côtoyant des régions qui suivraient la voie du développement et de la croissance à tout prix. Les territoires et les régions seraient encore plus différenciés, ce qui ne ferait qu'attiser les tensions ou la tentation d'un repli sur soi localiste. Enfin, le développement durable exige des changements qui demandent des efforts et des investissements. Sans coordination, sans partage des efforts, sans confiance réciproque entre acteurs et institutions, certains peuvent être tentés de faire défection puisqu'une bonne part des effets d'une politique de développement durable concerne le maintien et l'enrichissement de biens publics, ce que sont de nombreux biens environnementaux.

On peut comparer ces scénarios à la lumière des trois piliers (principes ou critères, selon le contexte) du développement durable (efficacité économique, intégrité écologique et équité sociale, que nous résumons par: économie, écologie et équité) et construire une matrice dans laquelle chaque scénario est, qualitativement, mais selon une échelle ordinale, évalué (tableau 6). 
L'échelle ordinale comprend cinq catégories en ordre décroissant : $++|+|+-/-/--$. Cette matrice cherche à équilibrer les meilleures intentions des scénarios avec leurs effets les plus probables. Elle reste un jugement porté sur des tendances complexes, mais un jugement raisonné et alimenté par les pratiques actuelles, soit déjà bien implantées ou en phase d'expérimentation.

Tableau 6 - Les scénarios d’aménagement à la lumière du développement durable

\begin{tabular}{|c|c|c|c|}
\hline Scénarios/Critères & $\begin{array}{c}\text { Poussée } \\
\text { développementaliste }\end{array}$ & Repli réglementaire & $\begin{array}{c}\text { Pari du } \\
\text { développement durable }\end{array}$ \\
\hline Économie & ++ & - & + \\
\hline Écologie & - & +- & ++ \\
\hline Équité & - & + & + \\
\hline
\end{tabular}

Le premier scénario cote, si on peut dire, très fort sur le critère économique, plus faible sur l'environnement et plutôt faible sur l'équité, du moins dans une première étape, à cause des avantages qui peuvent s'accumuler dans les régions "gagnantes ». Certains croient toutefois que le développement produit, avec un certain décalage, une meilleure protection de l'environnement, car les pays riches ont plus de moyens et de ressources à leur disposition pour mener avec efficacité une action pour la protection de l'environnement. Cette évolution temporelle a été nommée « courbe environnemental de Kuznets », mais les études comparatives donnent des résultats contradictoires ${ }^{13}$. Le second présente un profil différent : l'équité sociale est fortement accrue, la qualité de l'environnement est maintenue, voire légèrement améliorée, mais ses pratiques peuvent devenir un grand obstacle à l'économie. Enfin, le dernier, le pari du développement durable, même si l'on souhaite que, sur les trois critères, il soit gagnant partout, peut prendre, à la condition d'éviter le repli localiste, la forme suivante : l'économie progresse, mais à un rythme plus lent que dans le premier scénario; l'environnement est nettement amélioré et l'équité sociale aussi, du moins face aux risques environnementaux.

Ces scénarios sont évidemment des vues de l'esprit. Ils ne sont pas des prédictions ni même des projections, Mais ils sont tous plausibles avec plus ou moins de certitude. La méthode de production des scénarios accentue la cohérence interne de chacun, tout en augmentant les différences entre eux. La réalité se présente plutôt sous la forme d'une combinaison d'éléments appartenant à différents scénarios. Ils aident toutefois à préciser les enjeux, les voies possibles, y compris celles qu'on aimerait bien éviter. Ils préparent, espère-t-on, à prendre de meilleures décisions publiques. Enfin, ils permettent aux acteurs sociaux et aux participants de mieux communiquer entre eux et, peut-être, de saisir tout le mérite d'une participation délibérative sur les choix d'avenir. Pour reprendre les termes de Mannheim, ils sont une combinaison d'idéologie, de préférences, de valeurs et d'intérêts bien ancrés, et d'utopie, d'alternatives envisageables et réalisables.

\section{Notes et références}

Louis Guay est professeur au Département de sociologie de l’Université Laval.

2 Nadeau, N. et L.A. Sandberg (2003). « To Have your Cake and Eat it too ? Utility, Ecology, Equity and Québec's New Forest Act, 2001 » Cahiers de géographie du Québec, vol. $47, n^{\circ} 132$.

3 Beatley, T. (2000), Green Urbanism, Washington, Island Press; Vivre en ville (2001). Vers des collectivités viable, Sillery, Septentrion; Rabinovitch, J. et J. Lutman (2004). «Urban Planning in Curitiba », dans S.M. Wheeler et T. Beatley (dir.), The Sustainable Urban Development Reader, Londres, Routledge, p. 237-248; Hsiao, H-HM. et H.J. Liu (2004). « Collective Action toward a Sustainable City: Citizens' Movements and Environmental Politics in Taipei », dans S.M. Wheeler et T. Beatley (dir.), op.cit., Londres, Routledge, p. 259-274.

4 Wakernagel, M. et W. Rees (1999). Notre empreinte écologique, Montréal, Éditions Écosociété.

5 Aubertin, C. et al. (1999). « La construction sociale de la question de la biodiversité », Natures, Sciences et Sociétés, 
vol. 6, p. 7-19; Guay, L. (2002b). « The Science and Policy of Global Biodiversity Protection », dans P. Le Prestre (dir.), Governing Global Biodiversity: The Evolution and Implementation of the Convention on Biological Diversity, Aldershot, UK, Ashgate, p. 207-231.

6 Dearden, P. et J. Dempsey (2004). «Protected Areas in Canada : Decade of Change », The Canadian Geographer/ Le géographe canadien, vol. 48, p. 225-239.

7 Polèse, M. et R. Shearmur (2002). La périphérie face à l'économie du savoir, Montréal, INRS Urbanisation, Culture et Société et Institut canadien de recherche sur le développement du savoir :

www.inrs-ecs.uquebec.ca/F/inc/regions du $\mathrm{S} /$

8 Collin, J.-P. (2002). « La réforme de l'organisation du secteur municipal au Québec: la fin ou le début d'un cycle ? » Organisations et territoires, vol. 11, n 3, p. 5-13.

9 Slocombe, D. S. et P. Dearden, (2002). « Protected Areas and Ecosystem-based Management », dans P. Dearden et R. Collins (dir.), Parks and Protected Areas in Canada, Toronto, Oxford University Press, p. 295-320.
${ }^{10}$ Polèse, M. et R. Shearmur (2004). Le positionnement de Montréal par rapport à 11 autres agglomérations : entre perceptions et statistiques, Montréal, INRS Urbanisation, Culture et Société.

11 Hamel, P. (1999). « La consultation publique et les limites de la participation des citoyens aux affaires urbaines », Recherches sociographiques, vol. 40, p. 435-466; André, P., C.E. Delisle et J.-P. Revéret (2003). L'évaluation des impacts sur l'environnement : processus, acteurs et pratiques pour un développement durable, Montréal, Presses internationales polytechniques.

12 Functowicz, S.O. et J.R. Ravetz (1993). « Science for Postnormal Age », Futures, vol. 25, p. 739-755; Ravetz, J. (2003). « A Paradoxical Future for Safety and the Global Knowledge Economy », Futures, vol. 35, p. 811-826; Guay, L. (2005). «Controverses sociotechniques, participation et décisions publiques », dans L. Guay, P. Hamel, D. Masson et J.-G. Vaillancourt (dir.), Mouvements sociaux et changements institutionnels, PUQ, p. 375-418.

13 Day, K. et Q. Grafton (2001). « Economic Growth and Environmental Degradation », The Review of Economic Performance and Social Progress, p. 293-309. 


\section{Publicité}

«Centre de recherche sur le développement territorial " CRDT 